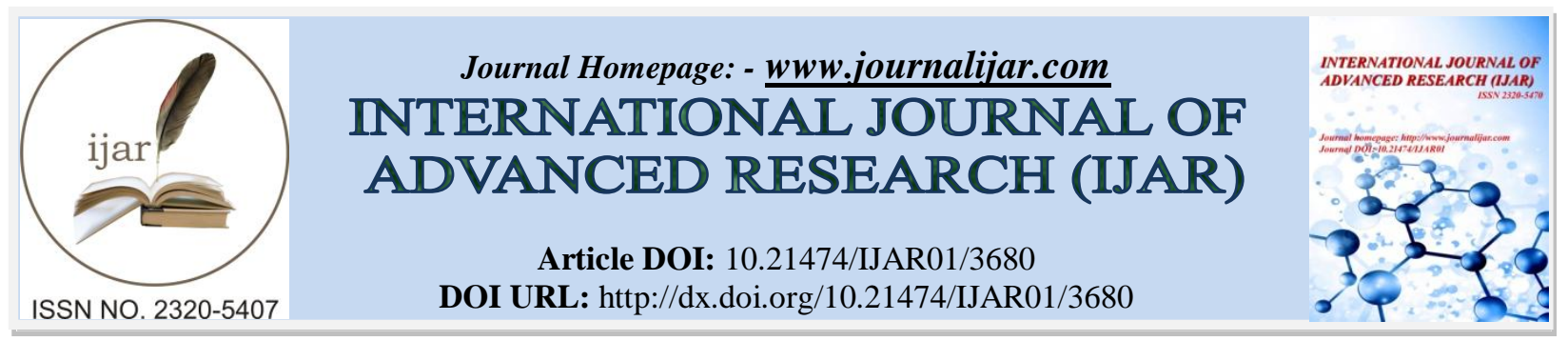

RESEARCH ARTICLE

\title{
REVIEW PAPER ON YAWNING DETECTION TO PREVENTS ROAD ACCIDENTS
}

Amruta. S. Kaitake ${ }^{1}$ and Mr. Vishnu Suryawanshi ${ }^{2}$.

1. Student, G.H Raisoni Institute of engineering and Technology Wagholi-Pune, India.

2. Faculty G.H Raisoni Institute of engineering and Technology Wagholi-Pune, India.

\section{Manuscript Info}

\section{Manuscript History}

Received: 12 January 2017

Final Accepted: 07 February 2017

Published: March 2017

\section{Key words:-}

Embedded vision algorithm, low complexity detection, Smart camera, vision-based measurement (VBM) yawning detection.

\begin{abstract}
For the safety purpose of drivers yawning detection is very important. There are many important applications of yawning in a drivers fatigue detection, humans well behavior, driving behavior and understanding the language of tongue disability person. It is observed that heavy vehicle drivers keep going continuously driving without giving a frequent rest period. Considering above applications, an automatic detection of yawning is one important system component. Uptil now yawning detection system does not satisfy the real time requirement which is having a high computational complexity and does not satisfies the challenges like facial obstruction, ease of implementation, accuracy and safety. Uptil now there is no any idea suggested or presented on vehicle side hardware. In this paper we will be reviewing hardware of engine start or stop control by using microcontroller.

In previous, for yawning detection two different algorithms are used such as for face viola-Jones and mouth detection contour activation algorithm. Previously presented systems are in position to recognize other persons faces while finding the biggest face in all of the frame that we are interested and other faces also considered; hence face search time is not optimized. By studying these disadvantages of traditional yawning detection method possible improvement algorithm is proposed.
\end{abstract}

Copy Right, IJAR, 2017,. All rights reserved.

\section{Introduction:-}

In earlier years the amounts of road accidents have been suddenly increased. These accidents because of continuous driving ability, not getting proper sleep. Especially in the transportation industry the heavy vehicle drivers keep continuously driving without giving a rest period. Due to this most of the accidents are occurs. Because of this it has become an area of great socioeconomic concern. Driver Fatigue is one of major reasons causing most fatal road accidents around the world.

The global status report on road safety 2013 shows that survey or information from 182 countries, calculated about $99 \%$ of the world's population. The report shows that around the world the number of deaths is due to the road accidents are high at 1.24 million per year. About 3,400 people die every day in world due to road accidents. Tens of millions of people are injured or disabled per year. Cyclist, pedestrians, children and the elderly are the most susceptible of road users. So it is very necessary to design such a prototype system which prevents road accidents by detecting driver's inattention, driver's drowsiness and giving a audio sound when yawning is detected. In this paper 
the aim is to develop a prototype system which detects yawning to prevent road accidents. This system includes a video camera either externally attached or the inbuilt camera in laptop which extracts video to detect face and mouth features. This system is non intrusive real time monitoring system. The programming for this is done in MATLAB 2013a using the image processing toolbox.

The purpose for so much attention towards drivers yawning detection is that suddenly increases road accidents and for the people's safety. Once fatigue has been detected, a number of activities we can performed for the safety purpose of person who drives the vehicle, mainly producing the persons alert voice, fluctuations in steering wheel or persons chair, showing text, providing large amount of oxygen for person himself.

There are many other applications for yawning detection is helpful in to be concerned with home health care technique. That proves yawning is one important method to detect the people's health information and modify their lifestyle. There are also more applications for yawning detection such as operator attentiveness to determining whether the operators such as heavy machines, nuclear reactor controls and monitors, and air traffic controllers in critical system is paying attention or not. Another application is that the system used in to determine the communication purpose of human being with language defect, particularly to detect false estimation.

For all of the above systems that require a natural investigation of involuntary intake, the value of technique is more important to create it cost effective. For that purpose VBM is very co-operative. VBM is a technique where a camera or optical sensor captures the photo from various scenes. Later this photo is used in process of yawning detection through manipulation component. This camera and manipulation component is called as intelligent camera. Because of this, VBM technique is most popular and useful now a day. Today the VBM, studied a experimental answer for the operations like investigation of persons natural or well being characteristics, for example facial expression or self propelled functional technique. Yawning detection system has number of important applications such as drivers fatigue detection, drivers behavior detection and also other automotive applications to prevents road accidents. Driving is a most common activity in people's everyday life. So it is very necessary to make driving safe for the peoples. The main reason to discover yawning detection method is significant increases the probability of car accidents. Most of the yawning detection system does not satisfy the real time requirement have a high computational complexity. So now a day's very few yawning detection system uses in a luxury cars to prevent car accidents. They do not have a sufficient accuracy. There are three main reasons why existing system is not used in our car system is

1. Computational complexity

2. Facial obstruction and

3. Lighting conditions

The main goal of this yawning detection method is lower the computational complexity and make this system safer to the person by studying real boundaries and can designing in actual intelligent cameras.

\section{Literature Survey:-}

In 2008 author described drivers fatigue detection based on trailing the jaws and to survey on controlling and recognising the involuntary intake. Author explained a technique to trace and trail the mouth cascade of classifiers is applied also the yawning images. After locating the mouth from face images using classifiers the SVM technique is applied to distinguish jaws also for to detect yawning then alert fatigue.

This is the non intrusive real time monitoring system. Many researches deal with yawning detection uses the geometric feature of the mouth techniques, but there are some disadvantages of that technique that is the left and right mouth corners are important feature point .But in this lip position are difficult to detect at that time the lips move, then lip detection makes more difficult. Also, geometric features liable to pose and have more difference for individual. This paper author used cascade of classifiers to train and trail with the help of viola-Jones. Then SVM is used for data classification.

After studying this paper we have conclude that, because of using SVM (support vector machine) computational cost is reduced and provide better generalization. There is disadvantage, two algorithms is used, hence increases computational time and this viola-Jones technique is not efficient to run in a real time on smart cameras if implemented as above that using cascade of classifiers [1]. 
In 2009 author describes to locate and tracking the drivers mouth using two CCD cameras. This is the real time monitoring system. Camera A is fixed; it is used to locate drivers head position. Camera B is movable or controllable, which is used to locate the mouth and extract the mouth information. In this first collecting all the videos from camera A and using image processing algorithm locate the drivers face. Then second with the help of face position in camera A shoot driver head position all the time by sending command to camera B through serial port. Then to detect and track driver's mouth in camera B, use haar- like features. Then at last by comparing the ratio of mouth height and width, yawning is detected.

After studying this paper we have concludes that, through this method resolution ratio of driver's mouth is higher than single camera. Also the feature information is higher accuracy. There is some disadvantage of this method, once the position between the driver or two cameras is changed, and then the relation between angle control volume and pixel control volume is also changed. So this technique is not intelligent, it needs some future work [2].

Authors Dr. Sheifali Gupta, Er. Garima describes a prototype system for detection of driver's drowsiness to prevent road accident. This system is non intrusive real time monitoring system. This system detects facial features and extracts video with the help of a video camera either externally attached or inbuilt camera in laptop. Eye closure and yawning is detected followed by alert. In this programming is done with the help of MATLAB 2013a with the help of computer vision toolbox.

After studying this paper we have concluded that, the aim of this paper is to prevent the road accidents by detecting eye closure and involuntary intake of drivers. A 1.3 Mp video camera used to capture the real time videos and for processing. In this the vision based system is used because of its accuracy and non intrusiveness. Only the disadvantage is that, a night vision camera is not used to capture the images in dark or dim light to increase the usability [3].

Narender Kumar, Dr. N.C. Barwar Presented eye blinking and yawning detection system in real time for monitoring drivers drowsiness. This system is the non intrusive real time monitoring system. In this system if the drivers eyes are remains closed for certain period of time and his mouth remains open to yawning then driver is said to be drowsy and then alarm is sounded. In this the detection of facial features viola Jones algorithm is applied and for mouth detection contour activation algorithm is used to find the contour of the lips.

After studying this paper we have concluded that, this system was tested in different light conditions for different subjects that are without eye glasses, with eye glasses, without moustache and with moustache. In case of females there is also same set up, programming, webcams for night and late experiments. Without eye glasses eye blink rate detected more accurate. Only the disadvantage is that different algorithm is used for face and mouth detection, so not easy to implement and uses OpenCV method in that considering the biggest face we are interested, also other faces are considered, hence face search time is not optimized [4].

In 2016 article author proposes yawning detection is important application in driving behaviour monitoring. In this author used to either faces or jaws detection very efficient algorithm that is viola-Jones algorithm. Later used back projection theory to determine yawning. The author used in yawning detection method for implementing and testing smart embedded camera is used. This is the real time monitoring system.

After studying this paper we have concluded that, by comparing existing method this system gives correct yawning detection rate. In this the camera is installed on the dash board of the car or in front mirror. The result of face and mouth detection also the involuntary intake or tiredness alarm indication is display on monitor. Only the disadvantage is that the author is not address the challenge of lighting condition, safety, facial obstruction. They only implementing up to detection of yawning, there is no further implementation on hardware side for safety purpose. They only improve computational complexity [5]. 


\begin{abstract}
Algorithm used:-
Face detection algorithm

Comparing to previous system this yawning detection method is explaining the structure of a quick or recapture, well organized face investigation algorithm known as viola-Jones algorithm. Firstly in face investigation method, each and every information given by prepared facial characteristics of every lump can be removed or reserved mainly five different folders in intelligent camera for maintains the calculating period. After some time for the investigation algorithm this folders which are answer of prepared facial characteristics are applied. As related to that same direction, this controlling method uses store costs rather than preparing or making division or using whole photo for searching facial characteristic at starting.
\end{abstract}

Every folder has above sets of information.

a. Facial characteristic point

b. Facial characteristic costs

c. Facial characteristic load

d. Perform division

In the face investigation, for viola-Jones algorithm it is possible nothing but since the different persons are multiple face dimensions, rather since the people have capability to near or taking out against the camera in related period, according to bigger or shorter face dimensions. For that purpose facial characteristic is necessary to measured.

One of important advantage of this yawning detection system, by comparing the Open CV method of viola-Jones, this method ends detecting the different face later on searching the primary face of every structure that is needed. Specially face with original topic, so remaining faces not studied. Hence face investigate period is saved. The face which is any time nearest to the camera this face is investigate primary in this method. The other persons faces is not considered and they are not large than the original persons face.

The diagram 1 shows instances for the vehicle riding situation, in that persons face and mouth investigate correctly. Also, for storing prepared facial characteristic in folders is help straightly through camera and ignoring prepared face investigation, the effect of this method is gains the rate of face investigation step and the capability of controlling method.

\title{
Mouth detection Method:-
}

After detecting the face, the down partial face is selected for the goal detection area. This is the identical method like a face investigation. Later on searching the largest mouth in structure all the information nearest to mouth area and dimension send towards yawning detection.

Fig 1:-.Persons face and mouth searched between different peoples.

Fig 2:- Yawning sequence. 

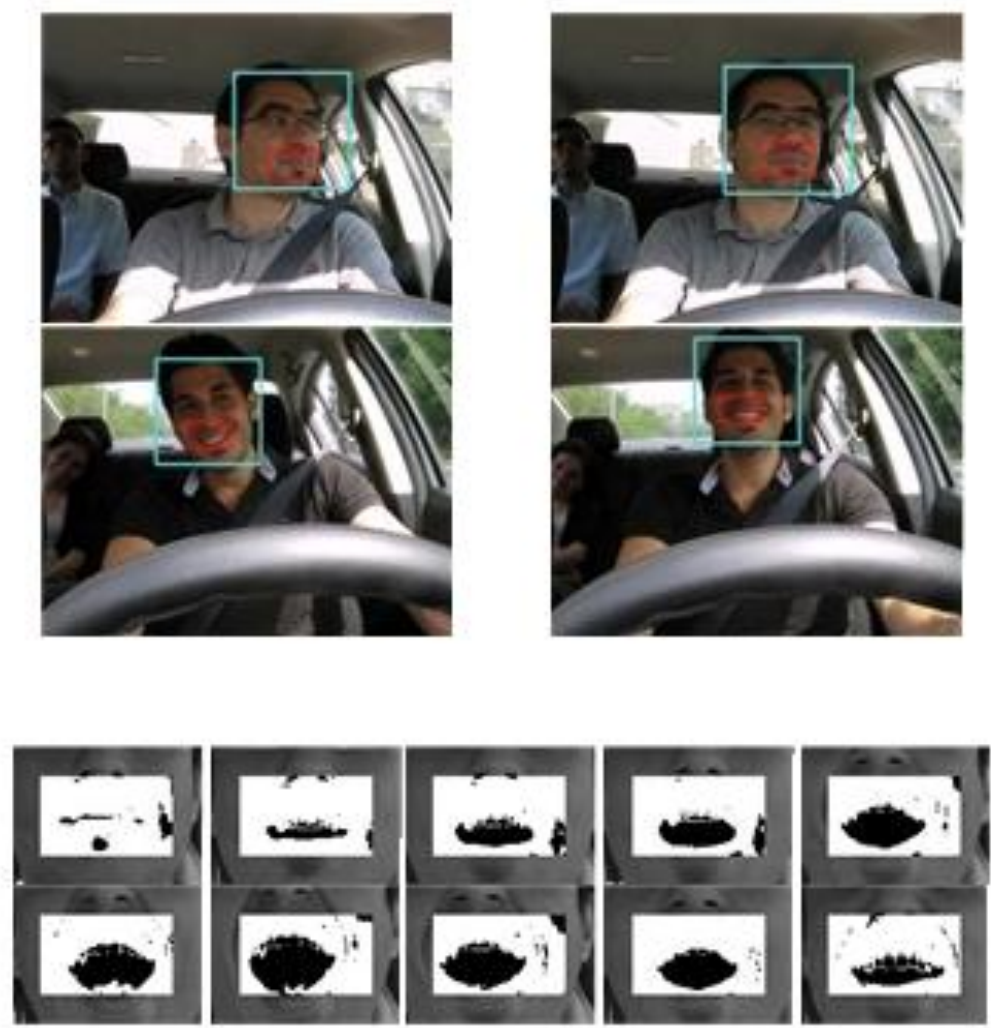

\section{Yawning detection Method:-}

The last part is yawning detection. The image is color image, so it is necessary to convert it into the gray scale image. The back-projection theory is used for that purpose.

After conversion of that grayscale photo the design take care not any involuntary intake in the structure. This is searched by comparing black and white elements. For that two rules are necessary to fulfilled.

a) Relation between black element in present folder and in relating folder should be larger than starting point.

b) Relation between black element in mouth area and white element in area surrounding to the mouth should be larger than second starting point.

Suppose these situations are fulfilled, according the design investigate specific folder shows involuntary intake and that procedure is copied for all of the later folders of motion picture.

\section{Proposed Work:-}

After studying literature survey and understanding need globally these papers are modified and a novel technique is build called yawning detection.

Below fig 3 displays the projected figure of yawning detection method. In this system the camera is installed in front of mirror or dash board in the car. MATLAB is used to build mathematical model of this system. In this viola-Jones algorithm is used for detecting face and mouth of driver. Viola-Jones algorithm is already installed in MATLAB. In hardware side microcontroller PIC16F877A is used along with other peripherals. PIC is family of Harvard architecture microcontroller made by micro chip technology.

Hardware side foot acceleration in that rack and pinion mechanism is used, which converts the rotary motion into the linear motion. Then amount of force is coming is passing on to the potentiometer, then that force is read on to the ADC. 
The microcontroller understands only digital logic that is either 0 or 1 also incoming voltage is AC; hence it needs to convert that AC voltage into DC volt. So ADC is used. The engine ON and OFF is depend on servo motor and in servo motor PWM that is pulse width modulation wave is inbuilt.

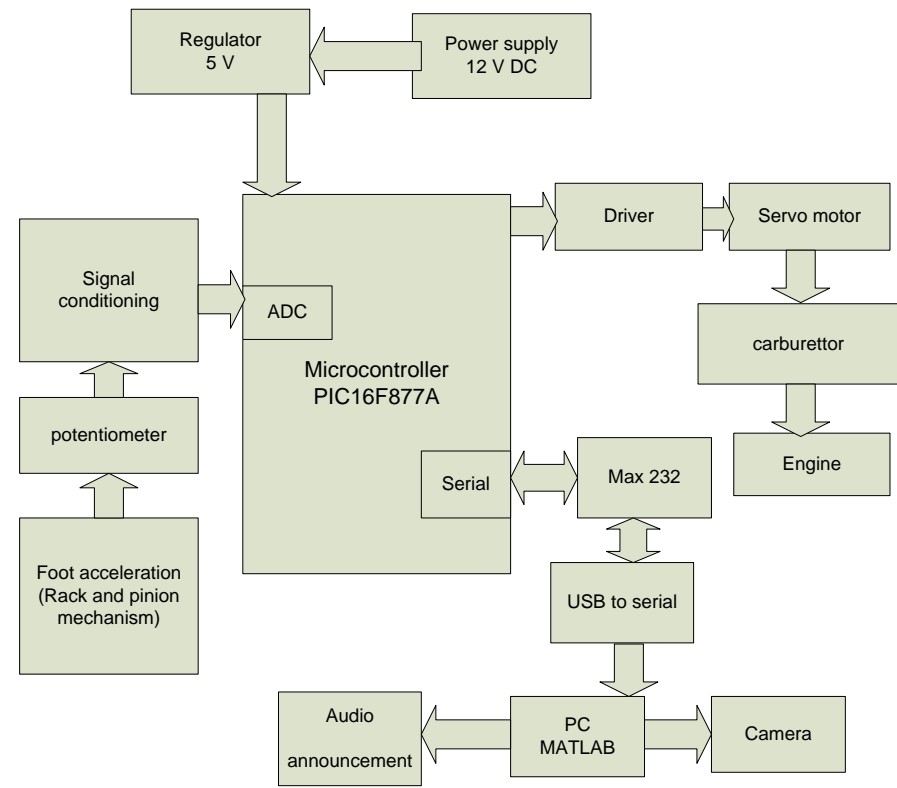

Fig 3:- projected yawning detection Method.

\section{Conclusion:-}

As we discussed until now yawning detection system, this system gives better accuracy, safety, easy implementation and reliability, reduce complexity, and increase efficiency. The previous papers have some disadvantages like no any implementation on vehicle side hardware and biggest candidate is the final detected mouth. In this paper we have reviewing hardware on PIC microcontroller and image processing part on MATLAB. For face and mouth detection this system monitors the condition based on delay and then takes decision. We will be reviewing hardware of engine start or stop using microcontroller. Also we will be taking care of acceleration of vehicle.

In car intex smart camera is placed in instrument panel, also in the foreground of the mirror. All the actions performed by the camera are running on embedded based system with help of this yawning detection method. To create yawning detection method act on a computationally limited platform \& address the problem of lighting condition, facial obstruction much effort was made in allocating or improving algorithm and codes to act in actual period. Also do not need any complex hardware. 


\section{References:-}

1. Mona Omidyeganeh, Member, IEEE, Shervin Shirmohammadi, Senior Member, IEEE, Shabnam Abtahi,Aasim Khurshid, Muhammad Farhan, Jacob Scharcanski, Senior Member, IEEE , Behnoosh Hariri, Daniel Laroche, and Luc Martel "Yawning Detection Using Embedded Smart Cameras "IEEE TRANSACTIONS ON INSTRUMENTATION AND MEASUREMENT, VOL. 65, NO. 3, MARCH 2016

2. Narender Kumar Dr. N.C. Barwar "Detection of Eye Blinking and Yawning for monitoring Driver's Drowsiness in Real Time" IJAIEM Volume 3, Issue 11, November 2014

3. Shabnam Abtahi Behnoosh Hariri Shervin Shirmohammadi "Driver Drowsiness Monitoring Based on Yawning Detection" Issue 2014

4. Mandalapu Saradadevi Dr. Preeti Bajaj "Driver Fatigue Detection Using Mouth and Yawning Analysis" IJCSNS International Journal of Computer Science and Network Security, VOL.8 No.6, June 2008

5. Nawal Alioua Aouatif Amine Mohammed Rziza"Driver's Fatigue Detection Based on Yawning Extraction" Research Article International Journal of Vehicular Technology

6. Dr. Sheifali Gupta Er. Garima" Road Accident Prevention System Using Driver's Drowsiness Detection by Combining Eye Closure and Yawning "International Journal of Research (IJR) Vol-1, Issue-6, July 2014 ISSN 2348-6848

7. Lingling Li Yangzhou Chen Zhenlong Li "Yawning Detection for Monitoring Driver Fatigue Based on Two Cameras" Proceedings of the 12th International IEEE Conference on Intelligent Transportation Systems, St. Louis, MO, USA, October 3-7, 2009

8. V. P. Minotto, C. B. O. Lopes, J. Scharcanski, C. R. Jung, and B. Lee, “Audiovisual voice activity detection based on microphone arrays and color information," IEEE J. Sel. Topics Signal Process., vol. 7, no. 1,pp. 147-156, Feb. 2013

9. S. Abtahi, M. Omidyeganeh, S. Shirmohammadi and B. Hariri, "YawDD: A yawning detection dataset," in Proc. 5th ACM MultimediaSyst. Conf., Singapore, 2014, pp.24-28.

10. H. Zhou, Q. Tang, L. Yang, Y. Yan, G. Lu, and K. Cen, "Support vector machine based online identification through advanced flame monitoring," Fuel, vol. 117, pp. 944-951, Jan. 2014

11. G. Bradski and A. Kaehler, Learning OpenCV: Computer Vision with the OpenCV Library. Sebastopol, CA, USA: O’Reilly Media, Inc., 2008, Ch. 13, p. 509. 